\title{
Medicinal plants used by the Batak Toba Tribe in Peadundung Village, North Sumatra, Indonesia
}

\author{
MARINA SILALAHI ${ }^{1, \boldsymbol{v}}$, NISYAWATI ${ }^{2}$, DINGSE PANDIANGAN ${ }^{3}$ \\ ${ }^{1}$ Departement of Biology Education, Faculty of Education and Teacher Training, Universitas Kristen Indonesia. Jl. Mayjen Sutoyo No.3, Cawang, Jakarta \\ 13510, Indonesia, Tel./fax. +62-21-8009190, `email: marina.silalahi@uki.ac.id; marina_biouki@yahoo.com \\ ${ }^{2}$ Departement of Biology, Faculty of Mathematics and Natural Science, Universitas Indonesia. Jl. Lingkar UI, Depok 16424, West Java, Indonesia \\ ${ }^{3}$ Departement of Biology, Faculty of Mathematics and Natural Science, Universitas Sam Ratulangi. Jl. Kleak-Bahu, Manado 95115, North Sulawesi, \\ Indonesia
}

Manuscript received: 14 November 2018. Revision accepted: 28 January 2019.

\begin{abstract}
Silalahi M, Nisyawati, Pandiangan D. 2019. Medicinal plants used by the Batak Toba Tribe in Peadundung Village, North Sumatra, Indonesia. Biodiversitas 20: 510-525. Research of the medicinal plants by the Toba Batak ethnic has limited, even though the globalization and modernization resulted to degradation of the local knowledge. The objectives of this study were (i) documentation of medicinal plants used in the traditional therapies by the Batak Toba tribe of Peadundung Village, North Sumatra, Indonesia, and (ii) analysing the data by quantitative ethnobotanical tools such as use value (UV), cultural significance index (CSI), relative frequency of citation (RCF) and informant consensus factor (ICF) to determine the cultural importance of medicinal plants in order to develop a tool for their conservation. Semi-structured interviews with 41 identified respondents was the methodology employed for qualitative data collection. A total of 149 medicinal species of plants, belonging to 131 genera and 55 families, were recorded in the study which are used in the treatment of 21 categories of ailments. Plants with the highest UV were Eurycoma longifolia (UV=3.44), Curcuma longa $(\mathrm{UV}=2.67)$ and Zingiber officinale (UV=2.60). Eight species, namely Curcuma longa, Eurycoma longifolia, Allium cepa, Psidium guajava, Aleurites moluccanus, Piper betle, Citrus hystrix and Uncaria gambir were found to be having the highest RCF value of 1.00 . Eurycoma longifolia (CSI=126), Curcuma longa $(\mathrm{CSI}=112)$ and Zingiber officinale (CSI = 105) emerged as the culturally most significant medicinal plants. Thrush and aphrodisiac use categories received the highest ICF of 1.00 each because the informants agreed of using only a single species for each of these category. Eurycoma longifolia was used as an aphrodisiac whereas Averrhoa carambola was used against thrush. All these important and significant plants suffer the greatest harvesting pressure, hence their conservation should be given priority.
\end{abstract}

Keywords: Batak Toba, Cultural Significance Index, Eurycoma longifolia, Informant Consensus Factor, Relative frequency of citation, Traditional medicinal plants, Use Value

\section{INTRODUCTION}

Research on medicinal plants used by indigenous ethnic groups is very interesting and useful because it has led to the development of many important modern drugs (Cox 2000). The current modern pharmacopoeias contain about $25 \%$ of the drugs derived from plants while many others are synthetic analogues built on prototype compounds isolated from plants (Fabricant and Farnsworth 2001).The Batak is an indigenous ethnic group of Sumatra Island, comprising of five tribes often referred to as Batak Karo, Batak Pakpak, Batak Simalungun, Batak Toba and Batak Angkola-Mandailing (Bangun 2010). The Batak Toba is the largest tribe with maximum population widely distributed in the highland of Toba District, Samosir in the Toba Lake region, North Tapanuli, and Humbang Hasundutan. The Batak people use plants as staple foods, vegetables, fruits, construction materials, spices, colouring substances and medicine.

Since ancient times, the Batak tribes have been using plants for the treatment of various ailments or in their traditional therapies. They possess basic knowledge about the use of medicinal plants in traditional health-related practices and products, such as oukup (traditional steam bath) (Nasution 2009), kuning and parem (powder or liquid used for traditional massage) (Silalahi 2014), minak alun (oil for traditional massage) (Purba et al. 2016), tinuktuk (traditional concotion for maintaining good stamina) (Sujarwo et al 2014), etc. Some of the notable medicinal plants that have been used by the Batak include Eurycoma logifolia Jack., Etlingera elatior (Jack.) R.M.Sm, Areca catechu L., and Curcuma longa L. (Nasution 2009; Silalahi et al. 2015; Sujarwo et al. 2014). Some researchers found that elderly people possess more detailed knowledge of medicinal plants than the young people (Silalahi et al. 2015; Begossi et al. 2002). The existence of medicinal plants in nature and the life-long maintenance of local knowledge point to the necessity of conservation of both plants and culture. (Sujarwo et al. 2014; Menendez-Baceta et al. 2015). However, most of the traditional knowledge about plants and their uses is fast disappearing owing to various factors like socioeconomic and land use changes (Segorini et al. 2009; Homerverge et al. 2014), increasing use of modern pharmaceuticals (Caniago et al. 2008) and increasing access to and use of biomedical health care (Ragupathy et al. 2008). Besides the loss of traditional 
knowledge about medicinal plants, the loss of traditional ecological knowledge is considered as a major threat to the success of conservation of biological diversity (Keller et al. 2005; Ju et al. 2013; Xavier et al. 2014; Sujarwo et al. 2016).

Various authors have conducted studies on medicinal plants used by various ethnic groups in Sumatra, including the Minangkabau (Ardan 2000), Rejang (Darnaedi 1999), Malay (Mahyar et al. 1991; Grosvenor et al. 1995; Rahayu et al. 2000), Lahat (Harmida and Yuni 2010), Serampas (Hariyadi and Ticktin 2010), Batak Karo (Silalahi et al. 2013; Purba et al. 2016) and Batak Simalungun (Silalahi et al. 2015). But, no such studies have been conducted on the Batak Toba tribe. In addition, there are no quantitative ethnobotanical studies on the rich ethnomedicinal plant and cultural diversity of Sumatra. Such quantitative ethnobotanical studies have been used to compare the uses and the cultural importance of different plant taxa in local communities (Albuquerque et al. 2006; Camou-Guerrero et al. 2008; Guimbo et al. 2011), to evaluate which are the most important plants within a culture and to determine conservation requirements (Homerverge et al. 2014; Albuquerque et al. 2006; Guimbo et al. 2011; TorreCuadros et al. 2003) and immaterial cultural heritage (Camou-Guerrero et al. 2008; Sujarwo and Caneva 2016). In particular, quantitative indices, such as use value or UV (Prance et al. 1987; Phillips and Gentry 1993; Albuquerque et al. 2006; Camou-Guerrero et al. 2008; Guimbo et al. 2011), relative frequency of citation or RFC (CamouGuerrero et al. 2008; Tardío and Pardo-de-Santayana 2008; Homerverge et al. 2014) index of cultural significance or CSI (Camou-Guerrero et al. 2008; Helida et al. 2015; Sujarwo and Caneva 2016; Silalahi and Nisyawati 2018) and informant consensus factor or ICF (Homerverge et al. 2014; Xavier et al. 2014; Sujarwo and Caneva 2016) are highly relevant in quantitative ethnobotanical study. They provide comprehensive and comparable information about the use of medicinal plants including their uses, conservation and cultural value (Guimbo et al. 2011; Helida et al. 2015; Sujarwo and Caneva 2016).

The present study aims at (i) documenting medicinal plant uses in the traditional therapies practiced by the Batak Toba tribe, and (ii) employing the quantitative ethnobotanical parameters, such as UV, CSI, RCF, and ICF, to determine the cultural importance of ethnobotanically valuable plants in order to develop a tool for their conservation.

\section{MATERIALS AND METHODS}

\section{Study area}

The ethnobotanical research and collection of botanical samples was conducted between August and December 2015 in Peadundung Village, Humbang District, North Sumatra, Indonesia (Figure 1). The Pedundung village lies at $02^{\circ} 07^{\prime} 62^{\prime \prime} \mathrm{N}$ latitude and $098^{\circ} 31^{\prime} 69^{\prime \prime}$ E longitude, at an altitude of 400-645 m above the sea level, about $332 \mathrm{~km}$ from Medan, the capital of North Sumatra. The total area of the Peadundung village is $15,2 \mathrm{~km}^{2}(1.527 \mathrm{ha})$ and is inhabited by 888 people belonging to 204 households of the Batak Toba. They were the descendants of the Proto Malay, and have been living there since about 100-200 years ago (personal communitation of the chief of village). About $99 \%$ of the population in this village are farmers, practicing rubber tree (Hevea brasiliensis) agroforestry. Peadundung village has a tropical climate with bimodal seasonality of dry season from April to July and rainy season from Agustus to April. The average annual temperature varies from 25 to $30^{\circ} \mathrm{C}$.

\section{Data colection}

Information on traditional uses of plants was gathered from a total of 41 informants, consisting of 9 key informants and 32 general respondents, ranging in age from 31-80 years. These respondents were selected with purposive snowball sampling methods. Key informants consist of folk healers ( 4 persons), midwifes ( 2 persons), chief of village ( 1 person) and head of customs ( 2 persons). The head of custom is a ceremonial leader who comes from the royal line, but the chief of village is the village leader in state administration. Information on uses and diversity of medicinal plants was obtained from interviews using the semi-structured, in-depth and participative observation methods, following the existing ethnobotanical guidelines (Martin 1995; Alexiades and Sheldon 1996; Silalahi et al. 2015a).

Voucher specimens of traditionally used medicinal plants were collected by way of exploration in the yards, agroforests, gardens, secondary forests and primary forests. and supplied with notes on life forms (tree, shrubs, herbs, ferns), local names, parts used, treatment and drug preparation methods. The initial identification of voucher specimens undertaken in the field and was later confirmed by taxonomists at the University of Indonesia Herbarium, Depok and the Herbarium Bogoriense of the Indonesian Institute of Sciences (LIPI) at Cibinong, Bogor, Indonesia. The scientific names of the medicinal plants were verified online with www.the plantlist, 2017 (The Plantlist 2017).

\section{Data analysis}

Data were analyzed using qualitative and quantitative methods (Alexiades, 1996). Qualitative analysis used the descriptive statistics by grouping plants based upon usage category. In the present study, we compared the importance of each species using the following four indices: use value (UV), relative frequency of citation (RFC), cultural significance index (CSI) and informant consensus factor (ICF).

\section{Use Value (UV)}

The relative importance of each plant species known locally to be used as herbal remedy was expressed as the use value (UV), which was calculated using the following formula (Phillips 1996).

$$
\mathrm{UV}=\frac{\sum U}{n}
$$




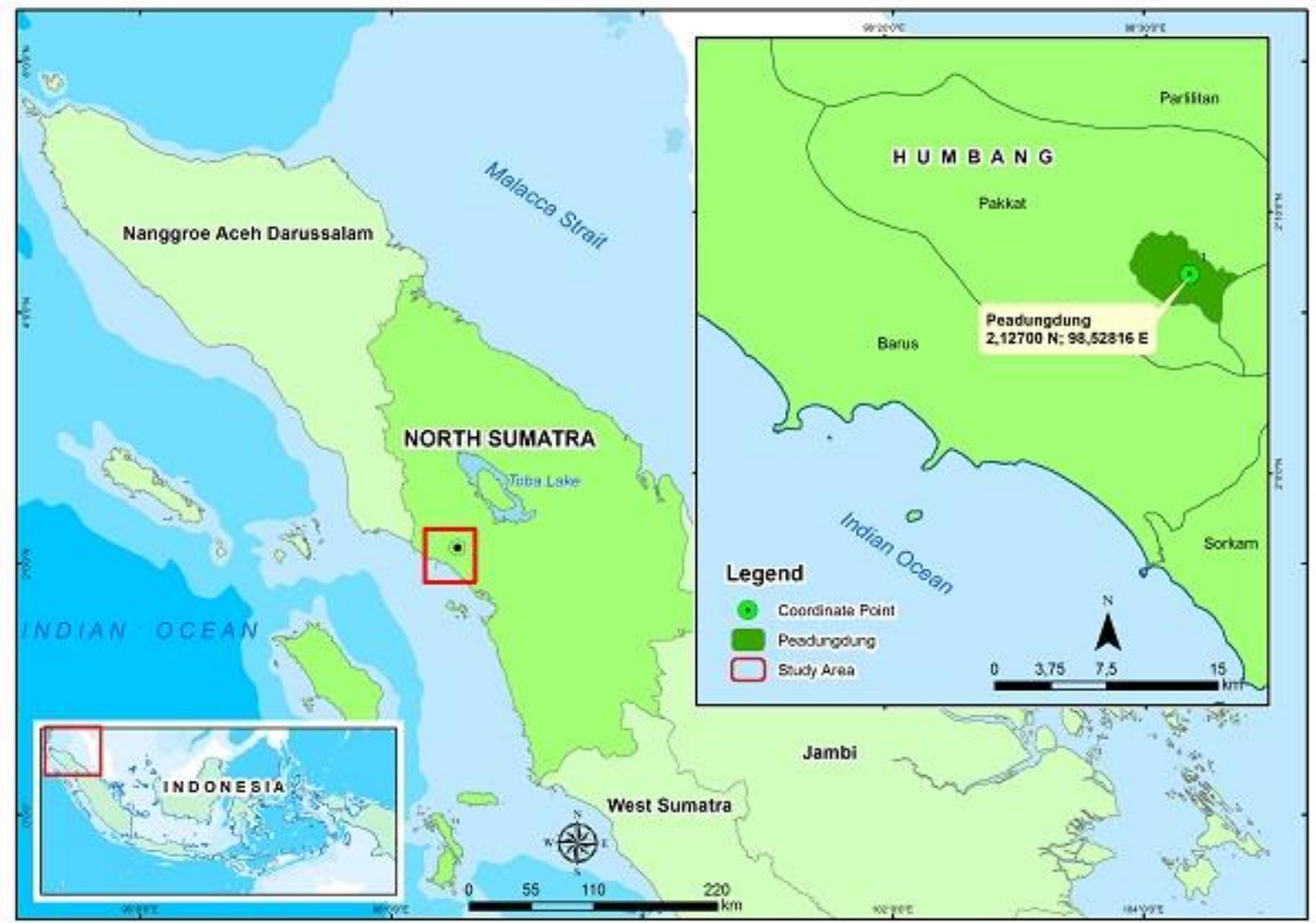

Figure 1. Peadundung village, North Sumatra, Indonesia (Map was reproduced from ArGIS 10.3)

The value of a species is UV, whereas the number of use-report cited by each informant is $\mathrm{U}$, and the total number of informants interviewed for a given plant is $n$.

\section{Relative Frequency of Citation (RFC)}

RFC is value of the a species by local communities (Tardío and Pardo-de-Santayana 2008).

$$
\mathrm{RFC}=\frac{F e}{\mathbb{N}}
$$

$\mathrm{Fc}=$ the number of informants mentioning the use of the species

$\mathrm{N}=$ informants $\mathrm{N}$

The value of RFC varies is $0-1$, if the value 0 mean nobody known of the use of plant, if the value 1 mean everybody know it uses.

\section{Cultural Significance Index (CSI)}

CSI is calculated using the through by Turner (1988) following formula:

$$
\operatorname{CSI}=\sum_{\mathbb{R}=1}^{ \pm(q x i x e) n i}
$$

The values of CSI is from 1 to $\mathrm{n}$, with $\mathrm{n}$ representing the last use described; the subscript $\mathrm{k}$ represents the value 1 through $\mathrm{n}$, consecutively. For each use given, $\mathrm{q}=$ quality value, $\mathrm{i}=$ intensity value, $\mathrm{e}=$ exclusivity value (Turner 1988).

\section{Informant Consensus Factor (ICF)}

The informant consensus factor (IFC) was used to see if there was agreement in the use of plants in the ailment categories between the plant users in the study area. The IFC was calculated by the following formula (Heinrich et al. 1998).

$$
I C F=\frac{N w r-N t}{N t-1}
$$

Nur is the number of use-reports for a particular ailment category and $\mathrm{Nt}$ is the number of taxa used for a particular ailment category by all informants.

\section{RESULTS AND DISCUSSION}

\section{Medicinal plants characteristics}

The present study documented the uses of 149 medicinal plant species belonging to 131 genera and 60 families for 21 different ailments. Among them, three 
species used were pterydophytes (Angiopteris avecta, Platycerium coronarium, Pteridium aquilinum). A total of 79 species $(53 \%)$ belong to 12 families, i.e., Asteraceae (10 species), Poaceae (10), Fabaceae (7), Solanaceae (7), Arecaceae (6), Myrtaceae (6), Zingiberaceae (6), Cucurbitaceae (5), Euphorbiaceae (5), Malvaceae (5), Rubiaceae (4), Araceae (4) and Lamiaceae (4) (Table 1). Out of 149 species recorded, the highest uses were recorded for abdominal pain (54), fever (45), injury (39) and fractures (19). The most common methods of preparation included boiling or soaking the plant parts in water, drying and grinding while the preferred route of administration was oral.

The medicinal plants, utilized for relieving abdominal pain and curing diarrhea, fever and malaria contain bitter substances, such as Eurycoma longifolia Jack, Clerodendrum chinense (Osbeck) Mabb, Lansium domesticum Corrêa, Durio zibethinus L., Dryobalanops aromatica C.F. Gaertn. and Artocarpus heterophyllus Lam. About 15 species of medicinal plants were also consumed as vegetables, 18 species as fruits, 14 as spices and 5 as staple foods. The species that were used as vegetables are Vigna unguiculata (L.) Walp., Cucumis sativus L., Cucurbita moschata Duchesne, Parkia speciosa Hassk. and Solanum melongena L. (Shrub). The carbohidrate resources such as: Clidemia hirta (L.) D. Don, Colocasia esculenta (L.) Schott., Melastoma malabathricum L. Pachyrhizus erosus (L.) Urb, Physalis angulata L. are used as wild fruits.

Analysis of medicinal plants based on their parts used as medicinal revealed that leaves are the highly used part (in 92 species), fruits are used in 20 species, stems or barks in 18 species and rhizomes in 6 species (Figure 2). In cases of some of the following species, more than one part may be used as medicine: Eurycoma longifolia Jack (leaves, stems, roots), Etlingera elatior (Jack.) R.M.Sm (stems and leaves) and Alpinia galanga L. (rhizomes and leaves). The species whose stems are used as medicinal materials included Lansium domesticum Corrêa, Artocarpus heterophyllus Lam, Durio zibethinus L. and Vatica pauciflora Blume. The species whose roots were used as medicinal materials are Eurycoma longifolia Jack, and Curculigo latifolia Dryand. ex W.T.Aiton.

The life forms of medicinal plants used by the Batak Toba consisted of herbs (72 species), trees (46 species), shurb (24 species) and climbers (7 species). The herbaceous species used by the Batak Toba included Blumea chinensis (L.) DC., Centella asiatica (L.), Emilia sonchifolia (L.) DC. ex DC., and Eryngium foetidum L.,). Notable examples for shrubs are Clibadium surinamense L., Sida rhombifolia L, Urena lobata L. and Melastoma malabathricum L. The recorded medicinal herbs were found mainly in disturbed plant communities while shrubs were found in advanced successional communities. In the present study, the medicinal plants were found in a wide range of habitats including home gardens, yards, fields, agroforests and forests. The majority of the plants were growing in wild (53\% species) and cultivated (35\%). 39 species of wild medicinal plants were otherwise considered as weedswhereas 40 are forest plants.
A total of 93 species $(55 \%)$ of the medicinal plants cited by 20 respondents $(50 \%)$ were easily found in the habitats around the village (Acorus calamus L. Centella asiatica (L.) Urb. and Areca catechu L.) or they were frequently used by the local communities (Blumea balsamifera (L.) DC., Hibiscus rosa-sinensis L. Lansium domesticum Corrê and Melastoma malabathricum L.). A total of 43 species were cited only by eight respondents (20\%) and they were Dryobalanops aromatica C.F.Gaertn., Imperata cylindrica (L.) Raeusch., Paspalum conjugatum P.J.Bergius, Physalis angulata L. and Platycerium coronarium (Mull.) Desv. Most of the local communities recognized Imperata cylindrica (L.) Raeusch. and Paspalum conjugatum P.J.Bergius as invaders or exotic plants.

\section{Quantitative analysis of medicinal plants}

Some the analytical tools can be used for a quantitative assessment of the cultural importance of individual medicinal plant species for Batak Toba community and also the degree of agreement among healers regarding the use of plants for specific disease categories. In this study, we compared the importance of each species using the following three indices: use value (UV), relative frequency of citation (RFC) and cultural significance index (CSI). Table 2 shows the top 20 plant species for each such index studied. The table shows a high variation among species emerging as important across the studied indices.

A total of 33 species, belonging to 31 genera and 20 families, share the top 20 positions of important medicinal plants based on their higher CSI, UV and RCF values.. They included 17 cultivated species, 12 wild species and 4 ruderal species. The wild and ruderal species should be given a priority in conservation, because they are more vulnerable and over-exploitated. They included Eurycoma longifolia Jack. Melicope glabra (Blume) T.G. Hartley, Rhodamnia sp., Styrax benzoin Dryand. and Timonius sericeus (Desf.) K.Schum. The local communities used them also as other economically useful commodities (Styrax benzoin Dryand), building materials (Melicope glabra (Blume) TG, Styrax benzoin Dry and Timonius sericeus (Desf.) K.Schum., Hartley) and firewood (Rhodamnia $\mathrm{sp}$ ). Styrax benzoin Dryand has a distinctive aroma that can have a relaxing effect and has been traded by the public as a medicinal ingredient hundreds of years ago.

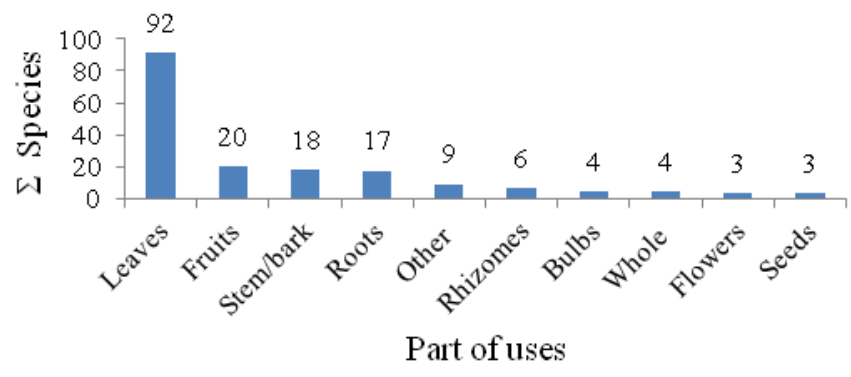

Figure 2. Medicinally useful parts and number of species in the medicinal practice of Batak Toba in North Sumatra, Indonesia. 
Table 1. The diversity of medicinal plants and their uses by the Batak Toba Tribe in Peadundung village, North Sumatra, Indonesia, along with quantitative values (UV, RCF and CSI)

\begin{tabular}{|c|c|c|c|c|c|c|c|c|c|}
\hline Family and botanical name & Life-form & Local name & Uses & $\begin{array}{c}\text { Mode of } \\
\text { application }\end{array}$ & $\begin{array}{c}\begin{array}{c}\text { Cultivation } \\
\text { status }\end{array} \\
\end{array}$ & Part used & UV & RFC & CSI \\
\hline \multicolumn{10}{|l|}{ Acanthaceae } \\
\hline Justicia gendarussa Burm.F. & Herb & Sipilit & Supranatural ailment, fever & Oral & Ruderal & Leaves & 1.30 & 0.59 & 30 \\
\hline Strobilanthes crispa Bl. & Shrub & Tepuringring & Abdominal pain & Oral & Wild & Leaves & 0.54 & 0.24 & 3 \\
\hline Stobilanthes sp. & Shurb & Pijor holing & Abdominal pain, injury, fever & Oral & Wild & Leaves & 1.91 & 0.29 & 75 \\
\hline \multicolumn{10}{|l|}{ Amaranthaceae } \\
\hline Celosia cristata $\mathrm{L}$. & Herb & Banda ulu & Fever & Oral & Ruderal & Leaves & 0.77 & 0.61 & 12 \\
\hline \multicolumn{10}{|l|}{ Amaryllidaceae } \\
\hline Crinum asiaticum $\mathrm{L}$. & Herb & Отри-отри & Fractures & & Ruderal & Bulbs & 1.00 & 0.68 & 30 \\
\hline Curculigo latifolia Dryand. Ex W.T.Aiton & Herb & Sukkit & Eye infection, headache & Oral & Wild & Roots & 0.53 & 0.22 & 6 \\
\hline \multicolumn{10}{|l|}{ Anacardiaceae } \\
\hline Gluta renghas $\mathrm{L}$. & Tree & Sipajal/sitorgom & Diabetes mellitus & Oral & Wild & Leaves & 0.30 & 0.20 & 3 \\
\hline Mangifera odorata Griff & & Ambasang & Diabetes mellitus, itch, diarrhea & Oral & Cultivated & Barks & 1.37 & 0.15 & 21 \\
\hline \multicolumn{10}{|l|}{ Annonaceae } \\
\hline Anona muricata $\mathrm{L}$. & Tree & Tarutung bulanda & Abdominal pain & Oral & Cultivated & Barks, leaves & 0.54 & 0.20 & 3 \\
\hline \multicolumn{10}{|l|}{ Apiaceae } \\
\hline Centella asiatica (L.) Urb. & Herb & Ampapaga & Fever, injury, abdominal pain & Oral & Wild & Leaves & 1.60 & 0.71 & 21 \\
\hline Eryngium foetidum $\mathrm{L}$. & Herb & Inggu & Partum, headache, supranatural disease & Inhalation & Wild & Leaves & 1.06 & 0.46 & 15 \\
\hline \multicolumn{10}{|l|}{ Apocynaceae } \\
\hline Alstonia pneumatophora Baker ex Den Berger & Tree & Goti & Ulcer, abdominal pain & Oral & Wild & Fruits & 1.07 & 0.22 & 36 \\
\hline Aquifoliaceae & & & & & & & & & \\
\hline \multicolumn{10}{|l|}{ Araceae } \\
\hline Acorus calamus $\mathrm{L}$. & Herb & Jarango & $\begin{array}{l}\text { Malnutrition, fever, headache, } \\
\text { supranatural disease }\end{array}$ & Inhalation, massage & Ruderal & Stem & 1.77 & 0.76 & 75 \\
\hline Alocasia macrorrhizos (L.) G.don & Herb & Lambuk & Itch & Massage & Wild & Stem & 0.30 & 0.15 & 4,5 \\
\hline Colocasia esculenta (L.) Schott. & Herb & Suhat & Itch & Massage & Cultivated & Stem & 0.38 & 0.17 & 3 \\
\hline Homalomena rubescens (Roxb.) Kunth & Liana & Langge & Fever, abdominal pain & Massage, oral & Wild & Stem & 0.53 & 0.17 & 4,5 \\
\hline Rhaphidophora nicolsonii P.C.Boyce & Liana & Gaol-gaol & Ulcer, fever & Massage, oral & Wild & Leaves & 1.30 & 0.41 & 48 \\
\hline \multicolumn{10}{|l|}{ Araliaceae } \\
\hline $\begin{array}{l}\text { Aralidium pinnatifidum (Jungh. \& De Vriese) } \\
\text { Miq. }\end{array}$ & Tree & Hau obang & Kidney disease & Oral & Wild & Leaves & 0.38 & 0.51 & 24 \\
\hline $\begin{array}{l}\text { Arthrophyllum diversifolium Blume } \\
\text { Arecaceae }\end{array}$ & Tree & Sipiturut & Abdominal pain & Oral & Wild & Stem & 0.38 & 0.20 & 3 \\
\hline Areca catechu $\mathrm{L}$. & Tree & Pining & $\begin{array}{l}\text { Diabetes mellitus, abdominal pain, } \\
\text { headache, fractures }\end{array}$ & Oral, massage & Cultivated & Roots, fruits & 1.53 & 0.61 & 48 \\
\hline Arenga pinnata (Wurmb) Merr. & Tree & Pola & Cough, fractures, diarrhea & Oral, massage & Ruderal & Roots, sap & 1.21 & 0.39 & 9 \\
\hline Calamus caesius Blume & Liana & Mallo & Fractures & Massage & Wild & Roots & 0.38 & 0.27 & 4,5 \\
\hline Daemonorops crinita Blume & Liana & Hotang & Fractures & Massage & Wild & Roots & 0.38 & 0.15 & 12 \\
\hline
\end{tabular}




\begin{tabular}{|c|c|c|c|c|c|c|c|c|c|}
\hline Cocos nucifera $\mathrm{L}$. & Tree & Harambir & Injury, headache, chiken pox & Massage, oral & Cultivated & Fruits, roots & 1.60 & 0.41 & 63 \\
\hline Salacca zalacca (Gaertn.) Voss & Tree & Salak & Diarrhea, fractures & Oral, massage & Cultivated & Sap & 1.67 & 0.15 & 30 \\
\hline \multicolumn{10}{|l|}{ Asteraceae } \\
\hline Ageratum conyzoides $(\mathrm{L}.) \mathrm{L}$. & Herb & Sibau-bau & Ulcer, fever, injury, diarrhea & Oral, massage & Wild & Leaves & 1.99 & 0.78 & 52 \\
\hline Blumea balsamifera (L.) DC & Herb & Langgumgum & Diarrhea, headache & Oral & Wild & Leaves & 1.53 & 0.78 & 49 \\
\hline Blumea chinensis (L.) DC. & Herb & Sirungkas & Fever, supranatural disease & Oral & Wild & Leaves & 0.60 & 0.44 & 9 \\
\hline Chromolaena odorata (1.) R.M.King \& H.Rob. & Shrub & Hau toba nalamis & Injury, abdominal pain, diarrhea & Oral, massage & Wild & Leaves & 1.61 & 0.90 & 60 \\
\hline Clibadium surinamense $\mathrm{L}$. & Shrub & Hau toba marrogon & Injury, abdominal pain, fever & Oral, massage & Wild & Leaves & 1.77 & 0.97 & 48 \\
\hline Emilia sonchifolia (1.) Dc. Ex dc. & Herb & Alum-alum & Itch, abdominal pain & Oral, massage & Wild & Leaves & 0.92 & 0.73 & 9 \\
\hline Gynura crepidioides Benth. & Herb & Nande rumah & Injury, headache, abdominal pain & Oral, massage & Wild & Leaves & 1.83 & 0.66 & 72 \\
\hline Mikania cordata (Burm.f.) B.L.Rob. & Herb & Anddor gila & Injury, abdominal pain, fever & Oral, massage & Wild & Leaves & 1.53 & 0.46 & 45 \\
\hline Vernonia arborea Welw. ex. O.Hoff. & Tree & Drasi & Fever & Oral & Wild & Leaves & 0.61 & 0.51 & 4,5 \\
\hline Vernonia sp. & Tree & Saur marnaik & Malnutrition & Steam-bath & Wild & Leaves & 0.38 & 0.29 & 4,5 \\
\hline \multicolumn{10}{|l|}{ Balsaminaceae } \\
\hline $\begin{array}{l}\text { Impatiens walleriana Hook.F. } \\
\text { Blechnaceae }\end{array}$ & Herb & Bunga pancur & Chiken pox, fever & Steam-bath & Wild & Leaves & 1.30 & 0.71 & 18 \\
\hline $\begin{array}{l}\text { Blechnum orientale } \mathrm{L} \text {. } \\
\text { Bombacaceae }\end{array}$ & Herb & Padung-padung & Ulcer, fever, malnutrition & Oral, steam-bath & Wild & Leaves & 0.75 & 0.51 & 9 \\
\hline Ceiba pentandra (L.) Gaertn. & Tree & Ponji & Fever, abdominal pain, malnutrition & Oral, steam-bath & Ruderal & Leaves & 1.38 & 0.39 & 39 \\
\hline $\begin{array}{l}\text { Durio zibethinus L. } \\
\text { Bromeliaceae }\end{array}$ & Tree & Tarutung & Fever, abdominal pain, malaria & Oral & Cultivated & Barks, leaves & 1.53 & 0.88 & 33 \\
\hline $\begin{array}{l}\text { Ananas comosus (L.) Merr. } \\
\text { Burseraceae }\end{array}$ & Herb & Honas & Cough & Oral & Cultivated & Fruits & 0.69 & 0.12 & 15 \\
\hline $\begin{array}{l}\text { Canarium pilosum A.W.Benn. } \\
\text { Caricaceae }\end{array}$ & Herb & Damar-damar & Itch & Massage & Wild & Leaves & 0.69 & 0.15 & 9 \\
\hline Carica papaya $\mathrm{L}$. & Herb & Botik & $\begin{array}{l}\text { Injury, abdominal pain, fever, } \\
\text { malaria }\end{array}$ & Oral, massage & Cultivated & Leaves, fruits & 2.12 & 0.90 & 60 \\
\hline $\begin{array}{l}\text { Caryophillaceae } \\
\text { Drymaria cordata (L.) Willd. Ex. Schult. }\end{array}$ & Herb & Hatiddi & Fever, headache & Oral & Wild & Leaves & 1.07 & 0.83 & 39 \\
\hline $\begin{array}{l}\text { Convolvulaceae } \\
\text { Ipomoea batatas }(\mathrm{L} .) \mathrm{Lam} . \\
\text { Costaceae }\end{array}$ & Herb & Gadong julur & Ulcer, fever & Oral, massage & Cultivated & Leaves & 1.38 & 0.32 & 36 \\
\hline $\begin{array}{l}\text { Costus spicatus (Jacq.) Sw. } \\
\text { Crassulaceae }\end{array}$ & Herb & Tabar-tabar & Cough & Oral & Ruderal & Rhizome & 0.38 & 0.15 & 3 \\
\hline $\begin{array}{l}\text { Kalanchoe pinnata (Lam.) Pers. } \\
\text { Cucurbitaceae }\end{array}$ & Herb & Hapal-hapal & Chiken pox, fever & Massage & Ruderal & Leaves & 0.83 & 0.51 & 36 \\
\hline Benincasa hispida (Thunb.) Cogn. & Herb & Gundur & Cough & Oral & Cultivated & Fruits & 0.46 & 0.30 & 18 \\
\hline Cucumis sativus $\mathrm{L}$. & Herb & Ancimun & Chiken pox, fever & Massage & Cultivated & Fruits & 0.77 & 0.54 & 4,5 \\
\hline Cucurbita moschata Duchesne & Herb & Jelok & Abdominal pain & Oral & Cultivated & Fruits & 0.45 & 0.20 & 4,5 \\
\hline Momordica charantia $\mathrm{L}$. & Herb & Pare & Hipertensi & Oral & Cultivated & Fruits & 0.62 & 0.29 & 4,5 \\
\hline $\begin{array}{l}\text { Sechium edule (Jacq.) Sw. } \\
\text { Cvatheaceae }\end{array}$ & Herb & Jipang & Hipertensi & Oral & Cultivated & Fruits & 0.46 & 0.54 & 4,5 \\
\hline $\begin{array}{l}\text { Cyathea contaminans (Wall. Ex Hook.) Copel. } \\
\text { Cyperaceae }\end{array}$ & Tree & Tandiang & Ulcer, fever & Massage & Wild & Leaves & 1.06 & 0.88 & 30 \\
\hline Cyperus rotundus $\mathrm{L}$. & Herb & Sitomu dalan & Fractures & Massage & Wild & Leaves & 0.46 & 0.20 & 3 \\
\hline
\end{tabular}




\begin{tabular}{|c|c|c|c|c|c|c|c|c|c|}
\hline \multicolumn{10}{|l|}{ Dennstaedtiaceae } \\
\hline Pteridium aquilinum (L.) Kuhn & Herb & Arsam & Fever, malnutrition & $\begin{array}{l}\text { Massage, steam- } \\
\text { bath }\end{array}$ & Wild & Leaves & 1.15 & 0.39 & 18 \\
\hline \multicolumn{10}{|l|}{ Dilleniaceae } \\
\hline Tetracera scandens (L.) Merr. & Liana & Andilo & Malnutrition, eye infection & Steam-bath, drop & Wild & Leaves & 0.38 & 0.78 & 12 \\
\hline \multicolumn{10}{|l|}{ Dipterocarpaceae } \\
\hline Dryobalanops aromatica C.F.Gaertn. & Tree & Hau hapur & Injury, malaria, abdominal pain & Massage, oral & Wild & Sap, leaves & 1.38 & 0.17 & 21 \\
\hline Vatica pauciflora Blume & Tree & Raru & Abdominal pain & Oral & Wild & Barks & 0.53 & 0.37 & 3 \\
\hline \multicolumn{10}{|l|}{ Euphorbiaceae } \\
\hline Aglaia argentea Blume & Tree & Sibalik angin & $\begin{array}{l}\text { Abdominal pain, supranatural } \\
\text { disease }\end{array}$ & Oral, massage & Wild & Leaves & 0.22 & 0.22 & 9 \\
\hline Aleurites moluccanus (L.) Willd. & Tree & Gambiri & $\begin{array}{l}\text { Diarrhea, ulcer, abdominal pain, } \\
\text { fever, itch }\end{array}$ & Oral, massage & Cultivated & Sap & 2.52 & 1.00 & 90 \\
\hline Manihot utilissima Pohl & Shrub & Gadong hau & Injury & Massage & Cultivated & Leaves & 0.53 & 0.17 & 12 \\
\hline Ricinus communis $\mathrm{L}$. & Shrub & Dulang bajora & Fever, abdominal pain & Massage, oral & Ruderal & Leaves & 0.68 & 0.78 & 27 \\
\hline Sauropus androgynus (L.) Merr. & Herb & Nasi-nasi & Partum, headache & Oral & Rderal & Leaves & 0.92 & 0.29 & 30 \\
\hline \multicolumn{10}{|l|}{ Fabaceae } \\
\hline Cassia alata $\mathrm{L}$. & Shrub & Galinggang & Itch & Oral & Wild & Leaves & 1.00 & 0.43 & 30 \\
\hline Mimosa pudica $\mathrm{L}$. & Herb & Podom-podom & Eye infection, kidney disease & Oral, drop & Wild & Roots & 1.30 & 0.17 & 12 \\
\hline Pachyrhizus erosus (L.) Urb. & Herb & Bangkuang & Hipertensi & Oral & Cultivated & Tuber & 0.61 & 0.20 & 12 \\
\hline Parkia speciosa Hassk. & Tree & Parira & Chiken pox dan itch & Massage & Cultivated & Fruits, leaves & 0.99 & 0.27 & 15 \\
\hline Archidendron pauciflorum (Benth.) I.C.Nielsen & Tree & Joring & Injury, abdominal pain & Massage, oral & Cultivated & Leaves, fruits & 1.30 & 0.46 & 46.5 \\
\hline Sp1. & Liana & Soit & Malnutrition & & Wild & Fruits & 0.53 & 0.41 & 9 \\
\hline Vigna sinensis (L.) Savi ex Hausskn. & Herb & Dali tanduk & Cough, injury & Oral, massage & Cultivated & Leaves & 0.92 & 0.17 & 12 \\
\hline \multicolumn{10}{|l|}{ Gleicheniaceae } \\
\hline Gleichenia linearis (Burm. F.) C.B. Clarke & Herb & Sampilpil & Fever, injury & Massage & Wild & Leaves & 1.38 & 0.56 & 33 \\
\hline \multicolumn{10}{|l|}{ Lamiaceae } \\
\hline Clerodendrum chinense (Osbeck) Mabb & Shrub & Sarang banua & Injury, diarrhea, abdominal pain & Massage, oral & Wild & Leaves & 1.83 & 0.56 & 21 \\
\hline Coleus amboinicus Lour. & Herb & Bangun-bangun & Fever, injury & Oral, massage & Cultivated & Leaves & 1.06 & 0.20 & 24 \\
\hline Ocimum americanum $\mathrm{L}$. & Herb & Bane-bane & $\begin{array}{l}\text { Fever, abdominal pain, supranatural } \\
\text { disease }\end{array}$ & $\begin{array}{l}\text { Oral, massage, } \\
\text { inhalation }\end{array}$ & Ruderal & Leaves & 1.54 & 0.41 & 39 \\
\hline Pogostemon cablin Blanco (Benth) & Herb & Nilam & Injury, abdominal pain & Massage, oral & Ruderal & Leaves & 1.30 & 0.27 & 36 \\
\hline \multicolumn{10}{|l|}{ Lauraceae } \\
\hline Cinnamomum cassia $(\mathrm{L}$.) J.Presl & Tree & Sitolu garis & Diarrhea, abdominal pain & Oral & Wild & Leaves & 0.68 & 0.21 & 6 \\
\hline Persea gratissima C.F.Gaertn. & Tree & Pokkat & Kidney disease & Oral & Cultivated & Leaves & 0.54 & 0.15 & 6 \\
\hline \multicolumn{10}{|l|}{ Liliaceae } \\
\hline Allium cepa $\mathrm{L}$. & Herb & Bawang merah & Fever, injury, abdominal pain & Oral, massage & Cultivated & Bulbs & 2.14 & 1.00 & 57 \\
\hline Allium sativum $\mathrm{L}$. & Herb & Bawang putih & Hipertensi, injury, abdominal pain & Oral, massage & Cultivated & Bulbs & 1.91 & 0.76 & 24 \\
\hline Cordyline fruticosa (L.) A.Chev. & Herb & Silinjuang & Supranatural disease, fever & Oral, massage & Ruderal & Leaves & 1.06 & 0.37 & 39 \\
\hline \multicolumn{10}{|l|}{ Malvaceae } \\
\hline Abelmoschus moschatus Medik. & Shrub & Purbajolma & Fractures, fever & Massage & Wild & Leaves & 1.07 & 0.46 & 45 \\
\hline Hibiscus rosa-sinensis L. & Shrub & Barbarsoma & Fever, chiken pox, malaria & Oral, massage & & Leaves & 2.30 & 0.90 & 57 \\
\hline Hibiscus sp. & Shrub & Ancilmong & Abdominal pain & & Wild & Leaves & 0.54 & 0.22 & 9 \\
\hline Sida rhombifolia $\mathrm{L}$. & Shrub & Sibagure & Injury, fractures & Massage & Wild & Roots & 1.45 & 0.44 & 42 \\
\hline Urena lobata $\mathrm{L}$. & Shrub & Sampelulut & Fever, fractures & Massage & Wild & Roots, flower & 1.38 & 0.41 & 36 \\
\hline
\end{tabular}




\begin{tabular}{|c|c|c|c|c|c|c|c|c|c|}
\hline \multicolumn{10}{|l|}{ Marattiaceae } \\
\hline Angiopteris evecta (G. Forst.) Hoffm. & Tree & Ingel-ingel & Ulcer, fever & Massage & Wild & Leaves, fruits & 0.46 & 0.49 & 12 \\
\hline \multicolumn{10}{|l|}{ Melastomataceae } \\
\hline Clidemia hirta (L.) D. Don & Shrub & Sanduduk & Injury, partum & $\begin{array}{l}\text { Oral, massage, } \\
\text { steam-bath }\end{array}$ & Wild & Leaves & 1.53 & 0.95 & 48 \\
\hline Melastoma malabathricum $\mathrm{L}$. & Shrub & Harimonting & Injury, abdominal pain & Oral, massage & Wild & Leaves & 1.53 & 0.37 & 45 \\
\hline Phyllagathis rotundifolia (Jack) Blume & Herb & Timba laut & Supranatural disease & Massage & Wild & Leaves & 0.69 & 0.32 & 9 \\
\hline \multicolumn{10}{|l|}{ Meliaceae } \\
\hline Lansium domesticum Corrêa & Tree & Latsat & Abdominal pain, diarrhea & Oral & Cultivated & Barks & 1.30 & 0.80 & 60 \\
\hline \multicolumn{10}{|l|}{ Moraceae } \\
\hline Artocarpus elasticus Reinw. Ex Blume & Tree & Torop & Abdominal pain, malaria, diarrhea & Oral & Wild & Barks & 0.76 & 0.17 & 15 \\
\hline Artocarpus heterophyllus Lam. & Tree & Pinasa & Cough, abdominal pain & Oral & Cultivated & Fruits, leaves & 0.84 & 0.20 & 30 \\
\hline Ficus sp. & Tree & Simarlayang-layang & Injury, diarrhea & Oral & Wild & Leaves & 0.45 & 0.15 & 6 \\
\hline \multicolumn{10}{|l|}{ Musaceae } \\
\hline Musa $\times$ paradisiaca $\mathrm{L}$. & Herb & Pisang sitabar & Injury, fractures & Massage & Cultivated & Roots, buds, sap & 1.38 & 0.66 & 36 \\
\hline \multicolumn{10}{|l|}{ Myrtaceae } \\
\hline Eugenia polyantha Barb. Rord. & Tree & Lomas & Diarrhea, abdominal pain, fever & Oral & Ruderal & Leaves & 1.30 & 0.95 & 52 \\
\hline Melaleuca leucadendra (L.) L. & Tree & Eucaliptus & Injury, abdominal pain & Oral, massage & Cultivated & Leaves & 0.68 & 0.34 & 12 \\
\hline Psidium guajava $\mathrm{L}$. & Tree & Attaboang & Abdominal pain & Oral & Cultivated & Leaves & 1.00 & 1.00 & 15 \\
\hline Rhodamnia sp. & Tree & Baja & Injury, toothaache abdominal pain & Oral, massage & Wild & Leaves, sap & 1.83 & 0.98 & 45 \\
\hline Syzygium sp. & Tree & Anggolam & Malnutrition & Steam-bath & Wild & Barks & 0.92 & 0.73 & 15 \\
\hline $\begin{array}{l}\text { Syzygium aromaticum (L.) Merril \& L.M. } \\
\text { Perry }\end{array}$ & Tree & Cengkeh & Cough & Oral & Cultivated & flower, leaves & 1.14 & 0.93 & 24 \\
\hline \multicolumn{10}{|l|}{ Nepentheceae } \\
\hline Nepenthes ampullaria Jack & Herb & Tahur-tahur tapongan & Injury & Massage & Wild & Leaves & 1.00 & 0.29 & 12 \\
\hline Nepenthes gracilis Korth. & Herb & Tahur-tahur manuk & Injury, eye infection, abdominal pain & Massage, drop, oral & Wild & Leaves & 1.60 & 0.34 & 36 \\
\hline \multicolumn{10}{|c|}{ 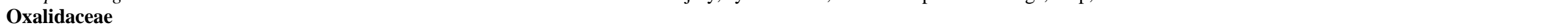 } \\
\hline Averrhoa carambola $\mathrm{L}$. & Tree & Balimbing & Thrush & Massage & Cultivated & Flower & 0.77 & 0.17 & 15 \\
\hline \multicolumn{10}{|l|}{ Pandanaceae } \\
\hline Pandanus amaryllifolius Roxb. & Herb & Pandan & Partum & Oral & Cultivated & Leaves & 0.61 & 0.37 & 12 \\
\hline \multicolumn{10}{|l|}{ Piperaceae } \\
\hline Piper betle L. & Herb & Napuran & Injury, partum, eye infection & Massage, drop, oral & Ruderal & Leaves & 1.91 & 1.00 & 75 \\
\hline Piper crocatum Ruiz. \& Pav. (Herb) & Herb & Napuran harangan & Fever, itch, malnutrition & Massage, drop, oral & Wild & Leaves & 0.52 & 0.20 & 45 \\
\hline Piper nigrum $\mathrm{L}$. & Herb & Lada & Headache, fractures & & Cultivated & Seeds & 1.38 & 0.63 & 42 \\
\hline \multicolumn{10}{|l|}{ Poaceae } \\
\hline Andropogon nardus $\mathrm{L}$. & Herb & Sangge-sangge holing & Malnutrition & Steam-bath & Cultivated & Whole & 0.99 & 0.46 & 18 \\
\hline Bambusa vulgaris Schrad. & Tree & Bulu & Malnutrition & Steam-bath & Wild & Roots, leaves & 1.15 & 0.41 & 3 \\
\hline Cymbopogon citratus (DC.) Staft. & Herb & Sangge-sangge & Malnutrition & Steam-bath & Cultivated & Whole & 1.44 & 0.51 & 33 \\
\hline Eleusine indica & Herb & Padang-padang & Chiken pox & Steam-bath & Wild & Roots & 0.53 & 0.20 & 12 \\
\hline Imperata cylindrica (L.) Raeusch. & Herb & Rih & Cough, abdominal pain & Oral & Wild & Roots & 0.53 & 0.12 & 3 \\
\hline Paspalum conjugatum P.J.Bergius & Herb & Duhut jau & Injury & Massage & Wild & Leaves & 0.77 & 0,12 & 12 \\
\hline Paspalum scrobiculatum $\mathrm{L}$. & Herb & Sanggar & Fractures & Massage & Wild & Roots & 0.46 & 0.12 & 3 \\
\hline Saccharum officinarum $\mathrm{L}$. & Herb & Tobu tawar & Injury, fractures & Massage & Cultivated & Stem & 0.76 & 0.12 & 21 \\
\hline Saccharum spontaneum $\mathrm{L}$. & Herb & Tolong & Fractures & Massage & Wild & Roots & 0.38 & 0.17 & 3 \\
\hline Vetiveria zizanioides (L.) Nash & Herb & Hapias & Abdominal pain & Oral & Wild & Leaves & 0.54 & 0.20 & 4,5 \\
\hline
\end{tabular}




\begin{tabular}{|c|c|c|c|c|c|c|c|c|c|}
\hline \multicolumn{10}{|l|}{ Polypodiaceae } \\
\hline \multicolumn{10}{|l|}{ Rosaceae } \\
\hline Rubus moluccanus Auct. & Shrub & Sihupi & Injury, diarrhea & Oral, massage & Wild & Leaves & 1.45 & 0.88 & 42 \\
\hline \multicolumn{10}{|l|}{ Rubiaceae } \\
\hline Coffea arabica $\mathrm{L}$. & Tree & Kopi & Itch & Massage & Cultivated & Seeds & 0.46 & 0.12 & 6 \\
\hline Myrmecodia tuberosa Jack & Herb & Pollang asar porgis & Itch, diabetes mellitus & Oral, massage & Wild & Stem & 0.15 & 0.07 & 3 \\
\hline Timonius sericeus (Desf.) K.Schum. & Tree & Simarbosi-bosi & Kidney disease, diarrhea & Oral & Wild & Leaves & 1.91 & 0.88 & 48 \\
\hline Uncaria gambir (Hunter) Roxb. & Shrub & Gambir & Abdominal pain & Oral & Ruderal & Sap & 1.00 & 1.00 & 30 \\
\hline \multicolumn{10}{|l|}{ Rutaceae } \\
\hline Citrus aurantiifolia (Christm.) Swingle & Tree & Utte bunga & Cough, itch & Oral, massage & Cultivated & Fruits, leaves & 0.99 & 0.88 & 27 \\
\hline Citrus hystrix DC. & Tree & Utte pangir & $\begin{array}{l}\text { Fever, supranatural disease, } \\
\text { malnutrition }\end{array}$ & $\begin{array}{l}\text { Oral, inhalation, } \\
\text { steam-bath }\end{array}$ & Cultivated & Fruits, leaves & 1.99 & 1.00 & 60 \\
\hline Melicope glabra (Blume) T.G. Hartley & Tree & Situkkol & $\begin{array}{l}\text { Injury, abdominal pain, malaria, } \\
\text { fever }\end{array}$ & Massage, oral & Wild & Leaves & 1.91 & 0.59 & 69 \\
\hline \multicolumn{10}{|l|}{ Sapindaceae } \\
\hline \multicolumn{3}{|l|}{ Solanaceae } & Abdominal pain, chiken pox, fever & Oral, steam-bath & Cultivated & Leaves, barks & 1.82 & 0.83 & 72 \\
\hline Capsicum frutescens $\mathrm{L}$. & Herb & Lasina & Eye infection, ulcer & Massage & Cultivated & Leaves & 1.06 & 0.41 & 27 \\
\hline Physalis angulata $\mathrm{L}$. & Herb & Pultak-pultak & Chiken pox, fever, hypertention & Steam-bath, oral & Wild & Whole & 1.53 & 0.17 & 57 \\
\hline Solanum melongena $\mathrm{L}$. & Shrub & Torung & Hypertention & Oral & Cultivated & Fruits & 0.62 & 0.24 & 4,5 \\
\hline Nicotiana tabacum $\mathrm{L}$. & Shrub & Timbaho & Abdominal pain, injury & Oral, massage & Cultivated & Leaves & 0.93 & 0.27 & 15 \\
\hline Solanum nigrum $\mathrm{L}$. & Shrub & Inggir-ingir & Hipertensi, headache & Oral & Wild & Fruits & 0.77 & 0.22 & 15 \\
\hline Solanum schiffnerianum Witasek & Herb & Inddot & Fractures & Massage & Wild & Leaves & 0.53 & 0.10 & 12 \\
\hline Solanum torvum Sw. & Shrub & Rimbang & Eye infection, fever, ulcer & Oral, massage & Ruderal & Leaves, fruits & 1.45 & 0.17 & 36 \\
\hline \multicolumn{10}{|l|}{ Sterculiaceae } \\
\hline Commersonia bartramia (L.) Merr. & Liana & Andor laut & Malaria, abdominal pain & Oral & Wild & Leaves & 0.54 & 0.22 & 12 \\
\hline \multicolumn{10}{|l|}{ Simaroubaceae } \\
\hline Eurycoma longifolia Jack & Tree & Tengku ali & $\begin{array}{l}\text { Fever, malaria, diarrhea, abdominal } \\
\text { pain, approdisiac }\end{array}$ & Oral & Wild & $\begin{array}{l}\text { Roots, stem, } \\
\text { leaves, seeds }\end{array}$ & 3.44 & 1.00 & 126 \\
\hline \multicolumn{10}{|l|}{ Styracaceae } \\
\hline Styrax benzoin Dryand. & Tree & Haminjon & $\begin{array}{l}\text { Supranatural disease, injury, } \\
\text { fractures, fever }\end{array}$ & Oral, massage & Cultivated & Sap, leaves & 1.69 & 0.80 & 57 \\
\hline \multicolumn{10}{|l|}{ Thymeleaceae } \\
\hline Aquilaria malaccensis Lam. & Tree & Alim & Abdominal pain & Oral & Wild & Sap & 0.77 & 0.10 & 4,5 \\
\hline \multicolumn{10}{|l|}{ Urticaceae } \\
\hline Leucosyke sp. & Herb & Hambing-hambing & Abdominal pain, eye infection & Oral, drop & Wild & Leaves & 0.92 & 0.15 & 12 \\
\hline Laportea decumana (roxb.) Wedd. & Shrub & Latong & Itch & Massage & Wild & Roots & 0.21 & 0.05 & 4,5 \\
\hline Leucosyke capitellata Wedd. & Herb & Simarihan-ihan & Malnutrition, eye infection & Steam-bath & Wild & Leaves & 0.93 & 0.37 & 30 \\
\hline \multicolumn{10}{|l|}{ Verbenaceae } \\
\hline Callicarpa sp. & Tree & Rittua & Fever, abdominal pain & Oral & Wild & Leaves & 0.91 & 0,20 & 6 \\
\hline \multicolumn{10}{|l|}{ Zingiberaceae } \\
\hline Alpinia galanga $\mathrm{L}$. Willd. & Herb & Halaos & Itch, malnutrition, diabetes mellitus & $\begin{array}{l}\text { Oral, steam-bath, } \\
\text { massage }\end{array}$ & Cultivated & $\begin{array}{l}\text { Rhizome, } \\
\text { leaves }\end{array}$ & 1.29 & 0.71 & 27 \\
\hline Curcuma longa $\mathrm{L}$. & Herb & Hunik & Cough, injury, itch, diarrhea, & Oral, steam-bath, & Cultivated & Rhizome & 2.67 & 1.00 & 112 \\
\hline
\end{tabular}




\begin{tabular}{|c|c|c|c|c|c|c|c|c|c|}
\hline Etlingera elatior (Jack.) R.M.Sm & Herb & Sihala dairi & $\begin{array}{l}\text { abdominal pain } \\
\text { Ulcer, fever, malnutrition, injury }\end{array}$ & $\begin{array}{l}\text { massage } \\
\text { Oral, steam-bath, } \\
\text { massage }\end{array}$ & Ruderal & Stem, leaves & 1.99 & 0.39 & 93 \\
\hline Hornstedtia leonurus (J.Koenig) Retz. & Herb & Sihala sisik & Malnutrition & Steam-bath & Wild & Stem, leaves & 1.00 & 0.39 & 30 \\
\hline Kaempferia galanga $\mathrm{L}$. & Herb & Hasihor & $\begin{array}{l}\text { Injury, malaria, supranatural } \\
\text { disease, partum }\end{array}$ & $\begin{array}{l}\text { Oral, steam-bath, } \\
\text { massage }\end{array}$ & Cultivated & Rhizome & 2.21 & 0.93 & 67 \\
\hline Zingiber purpureum Rosc. & Herb & Hunik burley & Diarrhea, abdominal pain, headache & Oral & Cultivated & Rhizome & 1.0 & 0.76 & 63 \\
\hline Zingiber officinale Rosc. & Herb & Pege & $\begin{array}{l}\text { Cough, diabetes mellitus, injury, } \\
\text { gastrointestinal disoldel }\end{array}$ & $\begin{array}{l}\text { Oral, steam-bath, } \\
\text { massage }\end{array}$ & Cultivated & Rhizome & 2.60 & 0.90 & 105 \\
\hline
\end{tabular}


Table 2. List of top 20 important medicinal plant species for the Batak Toba community, based on each of the three studied quantitative measures of relative importance (UV, RFC and CSI)

\begin{tabular}{|c|c|c|}
\hline Use Value (UV) & Relative Frequency of Citation (RFC) & Cultural Significance Index (CSI) \\
\hline Eurycoma longifolia Jack (3.44) & Eurycoma longifolia Jack (1.00) & Eurycoma longifolia Jack (126) \\
\hline Curcuma longa L. (2.67) & Curcuma longa L. (1.00) & Curcuma longa L. (112) \\
\hline Zingiber officinale Rosc. (2.60) & Piper betle L. (1.00) & Zingiber officinale Rosc. (105) \\
\hline Aleurites moluccanus (L.) Willd. (2.52) & Allium cepa L. (1.00) & Aleurites moluccanus (L.) Willd. (90) \\
\hline Hibiscus rosa-sinensis L. (2.30) & Aleurites moluccanus (L.) Willd. (1.00) & Piper betle L. (75) \\
\hline Kaempferia galanga L. (2.21) & Citrus hystrix DC. (1.00) & Gynura crepidioides Benth. (72) \\
\hline Allium cepa L. (2.14) & Uncaria gambir (Hunter) Roxb. (1.00) & Nephelium lappaceum L. (72) \\
\hline Carica papaya L. (2.12) & Rhodamnia sp. (0.98) & Melicope glabra (Blume) T.G. Hartley (69) \\
\hline Citrus hystrix DC. (1.99) & Clibadium surinamense L. (0.97) & Kaempferia galanga L. (67) \\
\hline Etlingera elatior (Jack.) R.M.Sm (1.99) & Eugenia polyantha Barb. Rord (0.95) & Zingiber purpureum Rosc. (63) \\
\hline Ageratum conyzoides (L.) L. (1.99) & Clidemia hirta (L.) D. Don (0.95) & Carica papaya L. (60) \\
\hline Piper betle L. (1.91) & Kaempferia galanga L. (0.93) & Citrus hystrix DC. (60) \\
\hline Allium sativum L. (1.91) & $\begin{array}{l}\text { Syzygium aromaticum (L.) Merril \& L.M. } \\
(0.93)\end{array}$ & Lansium domesticum Corrêa (60) \\
\hline Strobilanthes sp. (1.91) & Zingiber officinale Rosc. (0.90) & $\begin{array}{l}\text { Chromolaena odorata (L.) R.M.King \& } \\
\text { H.Rob. (60) }\end{array}$ \\
\hline Timonius sericeus (Desf.) K.Schum. (1.91) & Carica papaya L. (0.90) & Allium cepa L. (57) \\
\hline $\begin{array}{l}\text { Melicope glabra (Blume) T.G. Hartley } \\
(1.91)\end{array}$ & Hibiscus rosa-sinensis L. (0.90) & Physalis angulata L. (57) \\
\hline Gynura crepidioides Benth. (1.83) & $\begin{array}{l}\text { Chromolaena odorata (L.) R.M. King \& H. } \\
\text { Rob. }(0.90)\end{array}$ & Hibiscus rosa-sinensis L. (57) \\
\hline $\begin{array}{l}\text { Clerodendrum chinense (Osbeck) Mabb } \\
\text { (1.83) }\end{array}$ & Durio zibethinus L. (0.88) & Styrax benzoin Dryand. (57) \\
\hline Rhodamnia sp. (1.83) & $\begin{array}{l}\text { Cyathea contaminans (Wall. Ex Hook.) Copel. } \\
(0.88)\end{array}$ & Eugenia polyantha Barb. Rord. (52) \\
\hline Nephelium lappaceum L. (1.82) & Nephelium lappaceum L. (0.83) & Clibadium surinamense L. (48) \\
\hline
\end{tabular}

\section{Use Value (UV)}

The range of UV was between 0.15 - 3.44 with the mean value of $1.08( \pm 0.59)$. A higher UV indicated species that were considered most important by the Batak Toba, as shown by their number of use-reports in Table 1 . Three of the plants with the highest UV were Eurycoma longifolia Jack. (UV=3.44) having 5 use reports, Curcuma longa L. $(\mathrm{UV}=2.67)$ also having 5 use reports and Zingiber officinale Rosc. (UV=2.60) used in 4 disease categories. Eurycoma longifolia has been used to cure fever, malaria, diarrhea, abdominal pain, approdisiac, where some respondents only mention 3-4 benefits of them, so that the UV value is lower than 5. The respondent's knowledge about the use of medicinal plants is strongly influenced by age and ease of access to medicinal plants. However, $65.77 \%$ of all the recorded plants had more than one use report. For instance, Curcuma longa L. has been used to cure five of ailments (diarrhea, abdominal pain, cough, itch, injury). Such multiple uses demonstrated the importance of these plants as a part of the local cultural heritage.

\section{Cultural Significance Index (CSI)}

Based on the CSI value, medicinal plant species may be classified into five groups, as follows: species of very high significance (CSI> 200), species of high significance (CSI= 100-199), species of moderate significance (CSI= 20-99), species of low significance $(\mathrm{CSI}=5-19)$, and species of very low significance (CSI < 5) (Pieroni 2001). In the present study, the highest, the highest CSI value obtained was 126 and the lowest was 3. Accordingly, there were no species of very high cultural significance in the present study. 3 species were of high significance, 75 species were of moderate significance, 46 species were of low significance and 26 species were of very low significance (Figure 3). Eurycoma longifolia Jack., Curcuma longa L. and Zingiber officinale Rosc. were the top three species with high significance, whereas Cyperus rotundus L. (3), Imperata cylindrica (L.) Raeusch (3), Cucumis sativus L. $(4,5)$, and Laportea decumana (4,5) (Roxb.) Wedd. were examples for low significance species.

\section{Relative frequency of citation (RFC)}

The RFC based grouping of medicinal plants used by the Batak Toba is shown in Figure 4. The following eight species found to be having highest RCF (1.00 or 100\%): Allium cepa L., Aleurites moluccanus (L.) Willd., Citrus hystrix DC., Curcuma longa L. , Eurycoma longifolia Jack, Piper betle L., Psidium guajava L. and Uncaria gambir (Hunter) Roxb. Most of these plants were used in the treatment of abdominal pain (Aleurites moluccanus (L.) Willd., Allium cepa L., Curcuma longa L., Eurycoma longifolia Jack, Psidium guajava L. and Uncaria gambir (Hunter) Roxb.), for curing fever and supranatural purposes (Citrus hystrix DC. and Piper betle L.), and as aphrodisiacs (Eurycoma longifolia Jack). 


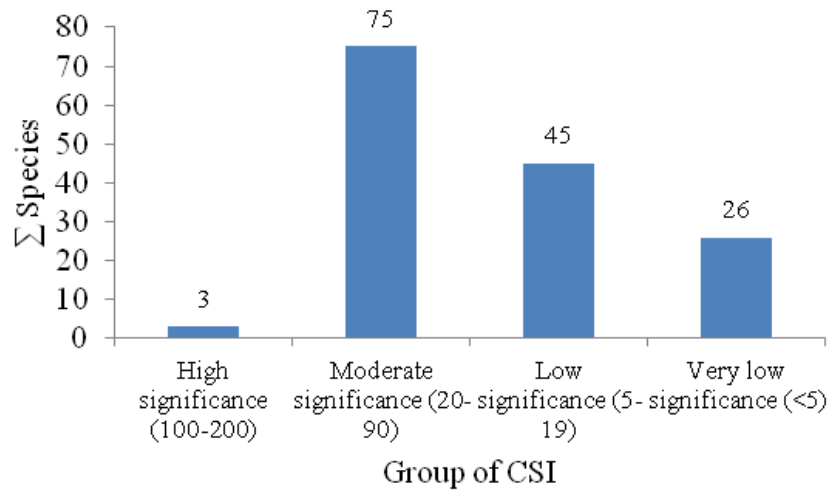

Figure 3. Categorisation of medicinal plant species used by Batak Toba, North Sumatra Indonesia, according to their CSI values.

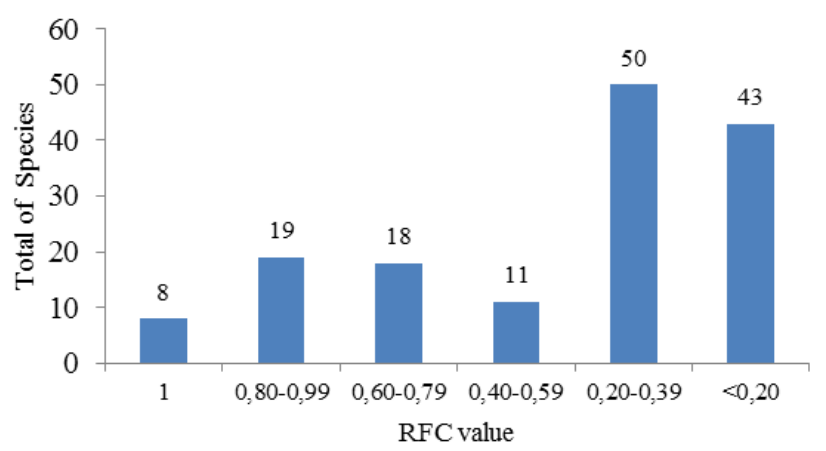

Figure 4. RFC value based grouping of medicinal plants used by Batak Toba of North Sumatra, Indonesia

\section{Informant Consensus Factor (ICF)}

The Batak Toba traditional healers generally used two or three plant parts for the preparation of medicines used in the treatment of single or multiple ailments. Use categories namely Thrush and aprodisiac have the highest ICF of 1.00 , but these categories ranked the lowest in the number of use reports ( 2 and 5 respectively) and number of species used ( 1 species, in each category). The use categories with more than 20 use reports were abdominal pain (134 use reports, 54 species), malaria (31 use reports, 9 species) and diabetes mellitus (17 use reports, 6 species) (Table. 3). The least agreement between the informants was observed in kidney disorders as indicated by the lowest ICF value of 0.400 , followed by fractures with a ICF of 0.469 . Thus, this study indicated that the degree of knowledge sharing by the users in the study area regarding the use of medicinal plants in the treatment of various ailments is low.

\section{Discussion}

The use of medicinal plants by local communities is influenced by various factors, such as pharmacological effectiveness, ecological availability and cultural factors (Menendez-Baceta et al. 2015). The cultural factors can shape the uneven distribution of medicinal knowledge across biogeographically similar regions and some such factors are language, social networks, meaning response (Vandebroek et al. 2004; Cocks and Dold 2006;
Table 3. Informant Consensus Factor (ICF) for different medicinal plant use categories

\begin{tabular}{|c|c|c|c|c|}
\hline $\begin{array}{c}\text { Ailment } \\
\text { Category }\end{array}$ & Nur & $\mathbf{N t}^{*}$ & ICF & $\begin{array}{c}\text { Frequently Used } \\
\text { Species }\end{array}$ \\
\hline Thrush & 2 & 1 & 1.000 & Averrhoa carambola $\mathrm{L}$. \\
\hline Aphrodisiac & 5 & 1 & 1.000 & $\begin{array}{l}\text { Eurycoma longifolia } \\
\text { Jack. }\end{array}$ \\
\hline Toothache & 5 & 2 & 0.750 & Rhodamnia sp. \\
\hline Malaria & 31 & 9 & 0.733 & $\begin{array}{l}\text { Eurycoma longifolia } \\
\text { Jack }\end{array}$ \\
\hline $\begin{array}{l}\text { Diabetes } \\
\text { Mellitus }\end{array}$ & 17 & 6 & 0.688 & Zingiber officinale Rosc. \\
\hline Diarrhea & 54 & 19 & 0.660 & $\begin{array}{l}\text { Uncaria gambir } \\
\text { (Hunter) Roxb.) }\end{array}$ \\
\hline Ulcer & 30 & 11 & 0.655 & $\begin{array}{l}\text { Rhaphidophora } \\
\text { nicolsonii P.C.Boyce }\end{array}$ \\
\hline Headache & 32 & 12 & 0.645 & Cocos nucifera $\mathrm{L}$. \\
\hline Partus & 15 & 6 & 0.643 & Acorus calamus L. \\
\hline Injury & 106 & 39 & 0.638 & $\begin{array}{l}\text { Melastoma } \\
\text { malabathricum L. }\end{array}$ \\
\hline $\begin{array}{l}\text { Supranatural } \\
\text { Ailment }\end{array}$ & 29 & 11 & 0.634 & Styrax benzoin Dryand. \\
\hline Fever & 119 & 45 & 0.627 & $\begin{array}{l}\text { Hibiscus rosa-sinensis } \\
\text { L. }\end{array}$ \\
\hline $\begin{array}{l}\text { Eye } \\
\text { Infection }\end{array}$ & 17 & 7 & 0.625 & Piper Betle L. \\
\hline $\begin{array}{l}\text { Abdominal } \\
\text { Pain }\end{array}$ & 134 & 54 & 0.602 & $\begin{array}{l}\text { Uncaria gambir } \\
\text { (Hunter) Roxb.) }\end{array}$ \\
\hline Chicken Pox & 21 & 9 & 0,600 & Physalis angulata $\mathrm{L}$ \\
\hline Cough & 21 & 11 & 0.565 & $\begin{array}{l}\text { Syzygium aromaticum } \\
\text { (L.) Merril \& L.M. } \\
\text { Perry }\end{array}$ \\
\hline Hypertension & 10 & 5 & 0.556 & Allium sativum $\mathrm{L}$. \\
\hline Itches & 32 & 18 & 0.528 & Cassia alata $\mathrm{L}$. \\
\hline Malnutrition & 32 & 16 & 0.516 & Acorus calamus L. \\
\hline Fractures & 33 & 18 & 0.469 & Sida rhombifolia $\mathrm{L}$. \\
\hline $\begin{array}{l}\text { Kidney } \\
\text { Disorder }\end{array}$ & 6 & 4 & 0.400 & $\begin{array}{l}\text { Aralidium pinnatifidum } \\
\text { (Jungh. \& De Vriese) } \\
\text { Miq. }\end{array}$ \\
\hline Total & 751 & 304 & & \\
\hline
\end{tabular}

Note: *A taxon may be reported in more than one use category. Nur: Number of Use-Reports, Nt: Number of Taxa, ICF: Informant Consensus Factor

Menendez-Baceta et al. 2015) and enviromental diversity (Eyssartier et al. 2008). The language is one of the frontiers that hinder the diffusion of local knowledge across linguistically distinct areas (Perales et al. 2005).

This study documented 149 medicinal plant species belonging to 56 families which are used for the treatment of 21 disease/use categories. The higher number of medicinal plant species documented in the current study when compared with an earlier study with another related tribe called Batak Phakpak (Silalahi 2006) shows that the Batak Toba has a richer tradition of medicinal plant use and therapy.But, the medicinal plant knowledge of Batak Toba is poorer in comparison to the Batak Karo tribe which uses 156 species (Silalahi et al. 2013) and Batak Simalungun tribe that uses 239 species (Silalahi et al. 2015). Despite the fact that all the three are named Batak tribes (Karo, Simalungun and Batak Toba) and they inhabit North Sumatra, they language and cultural differences. Some of 
the medicinal plants used by Batak Toba tribe are also used by the other Batak tribes residing in other sections of North Sumatra. They include Acorus calamus L., Ageratum conyzoides (L.) L., Citrus hystrix DC., Etlingera elatior (Jack.) R.M.Sm., Eurycoma longifolia Jack and Zingiber officinale Rosc. (Silalahi et al. 2015; Silalahi et al. 2013). However, some of the medicinal plants recorded in this study such as Melicope glabra (Blume) T.G. Hartley (situkkol), Timonius sericeus (Desf.) K.Schum (Simarbosibosi), Rhodamnia sp. (Baja) and Vatica pauciflora (Raru) are new reports with regard to healing of diarrhea and abdominal pain. The difference of species CSI is influenced by the level of knowledge, the particular cultural settings and the local conditions (Turner 1988; Pei et al. 2009; Helida et al. 2015).

In this research, quantitative ethnobotanical tools such as CSI, UV, RFC and the ICF were used to make the results more comprehensive to prioritize conservation of medicinal plants (Byg and Baslev 2001; Kvist et al. 2001; Dalle et al. 2004) and also to facilitate bioprospecting (Xavier et al. 2014; Silalahi et al. 2015). The values of RFC, UV and ICF are quantitave indicators that measure the cultural significance and importance of traditional medicinal plants (Thomas et al. 2009; Ong and Kim 2014; Menendez-Baceta et al. 2015; Sujarwo and Caneva 2015). The values of RFC, UV, and ICF are based on the respondents knowledge (Silva et al. 2006) whereas CSI is based on the analisys of researchers (Turner 1988; Hoffman and Timothy 2007).

Phillips and Gentry (1993) have develoved quantitative measure to know the species relative importance, which know as UV. UV has also been associated with issues of conservation, based on the idea that the most important species will suffer the greatest harvesting pressure (Albuquerque et al. 2006). Eurycoma longifolia Jack. (UV=3.44), Curcuma longa L. (UV=2.67, and Zingiber officinale Rosc. (UV=2.60) are the plants with the highest of UV in this study which indicates that these plants are considered most important as medicines by the Batak Toba tribe. UVs are high when there are many use-reports for a plant, implying that the plant is important, and low when there are few use-reports (Ong and Kim 2014). There are factors influencing the respondents knowledge of plants, important among others are age (Voeks 2007; Quinlan and Quinlan 2007; Guimbo et al. 2011), gender (Quinlan and Quinlan 2007; Camou-Guerrero et al. 2008; Guimbo et al. 2011), formal education (Voeks 2007; Quinlan and Quinlan 2007; Giovannini et al. 2011), use of biomedicines (Vandebroek et al. 2004), occupation (Quinlan and Quinlan 2007) and village level (Sujarwo et al. 2014). The level of formal education (Quinlan and Quinlan 2007; Giovannini et al. 2011; Sujarwo et al. 2014) and use of biomedicines (Vandebroek et al. 2004; Giovannini et al. 2011) have a negative correlation with the level of local knowledge; but age has a positive correlation with level local knowledge (Silalahi et al. 2015a). Number of medicinal plants known by women is generally higher than men (Camou-Guerrero et al. 2008; Guimbo et al. 2011). The CSI value depends on quality, intensity and exclusivity of species of the medicinal plants (Turner 1988; Silalahi et al. 2015a). This research showed that Curcuma longa L., Eurycoma longifolia Jack. and Zingiber officinale Rosc. has high cultural significance in the Batak Toba tribe, on the basis of their CSI values.

In the present study, plants used as aphrodisiac and in the treatment of thrush had the highest ICF of 1 each. A high value ICF indicates the agreement of selection of taxa between informants, whereas a low value indicates disagreement (Ragupathy et al. 2008; Xavier et al. 2014). ICF can thus be used to pinpoint particularly interesting species for the search of bioactive compounds (Canales et al. 2005). Thrush and aprodisiac have the highest ICF of 1.00 each because the informants agreed of using only a single species for each category. Eurycoma longifolia Jack is used as aphrodisiac, whereas Averrhoa carambola L. is used against thrush. High ICF values were also observed for use categories related to toothache, fever, malaria, diarrhea, diabetes, abdominal pain gastrointestinal disorder, etc. This finding suggested that there is a well-defined plant selection criterion for these use categories (Srithi et al. 2009; Silva et al. 2005). Cultural importance indices make it possible to quantify the role that a given plant plays within a particular culture, and CSI is used to evaluate and classify these plants according to their respective cultural significance (Pieroni 2001).

This study identified Eurycoma longifolia Jack., Dryobalanops aromatica C.F.Gaertn, Vatica pauciflora Blume and Melicope glabra (Blume) T.G. Hartley as having high overall use value, but according to our field data these plant species have a restricted distribution and a low abundance. Eurycoma longifolia Jack. has been phytochemically investigated by many researchers (Ang et al. 2000; Kuo et al. 2003; Chan et al. 2004; Farouk et al. 2007; Achmad et al. 2008; Talbott et al. 2013), but such studies are yet to be undertaken with Dryobalanops aromatica C.F.Gaertn, Vatica pauciflora Blume and Melicope glabra (Blume) T.G. Hartley.

Styrax benzoin Dryand is included in the 20 plants with the highest CSI but it is rarely found in the environment around. Styrax benzoin Dryand is indigenous in Sumatra island especially in Humbang and Tapanuli District (Kashio and Johnson 2001; Langenheim 2003; Lopez and Shanley 2004; Kusters and Belcher 2008), has been used by local communities in North Sumatra as an export commodity since the $8^{\text {th }}$ century (Backer and Bakhuizen van den Brink 1965; Boer and Ella 2001) is called Sumatra benzoin (Boer and Ella 2001; Kashio and Johnson 2001). Resin of Styrax benzoin Dryand in North Sumatera (Backer and Bakhuizen van den Brink 1965; Boer and Ella 2001), at the beginning taken by the local community from wild plants in forest (Kusters and Belcher 2008), and has been cultivated by the Batak ethnic since 200 years ago (Lopez and Shanley 2004).

It was summarized that the local communities of Batak Toba in Peadungdung village uses 149 medicinal plants belonging in 131 genera of 55 families to cure 21 type of ailment. A total number of the species having highest CSI, $\mathrm{UV}$, and RCF in top 20 plant species ranking was 33 species, belonging to 31 genera and 20 families. Medicinal plants with the highest recorded UV, CSI, RFC, and IFC 
were Eurycoma longifolia Jack, Curcuma longa L., and Zingiber officinale Rosc. Eurycoma longifolia Jack. and Styrax benzoin Dryand, Dryobalanops aromatica C.F.Gaertn, Vatica pauciflora Blume, and Melicope glabra (Blume) T.G. Hartley as having high overall use value but according to our field data, these plant species have a restricted distribution and a low abundance, so which species needs the protection and the ex situ and in situ conservation.

\section{ACKNOWLEDGEMENTS}

We would like to express our gratitude to local communities of Peadungdung villages, North Sumatra, Indonesia for the permission granted to us to carry out this research and also for their help in the field. We are also grateful to the staff of Herbarium Bogorience for their help in the identification of plants. Thanks to Kuswata Kartawinata for revising the English language version.

\section{REFERENCES}

Achmad SJ, Syah YM, Hakim EH, Juliawaty LD, Makmur L, Mujahidin D. 2008. Ilmu Kimia dan Kegunaan Tumbuh-tumbuhan Obat Indonesia. Institut Teknologi Bandung, Bandung. [Indonesian]

Albuquerque UP, Lucena RFP, Monteiro JM, Florentino ATN, Almeida CFCBR. 2006. Evaluating two quantitative ethnobotanical techniques. Ethnobot Res Appl 4: 51- 60.

Alexiades MN, Sheldon JW. 1996. Selected Guidelines for Ethnobotanical Research: A Field Manual. The New York Botanical Garden Press, New York.

Backer CA, van Den Brink B. 1965. Flora of Java (Spermatophyte Only) Vol. II. Angiospermae, Families 111-160. N. V. P. Noordhoff, Groningen.

Bangun P. 2010. Bataks Culture. in: Koentjaraningrat. Man and Culture in Indonesia. Djambatan, Jakarta. [Indonesia]

Boer E, Ella AB. 2001. Plant Resources of South-East Asia 18: Plants Producing Exudates. Prosea, Bogor.

Begossi A, Hanazaki N, Tamashiro JY. 2002. Medicinal Plants in the Atlantic Forest (Brazil): Knowledge, Use, and Conservation. Human Ecol 30: 3281-299.

Byg A, Baslev H. 2001. Diversity and use de palms in Zahamena, eastern Madagascar. Biodiversity and Conservation 10: 951-970.

Camou-Guerrero A, Reyes-García V, Martínez-Ramos M, Casas A. 2008 Knowledge and Use Value of Plant Species in a Rarámuri Community: A Gender Perspective for Conservation. Human Ecol 36: 259-272.

Caniago I, Siebert SF. 1998. Medicinal plant ecology, knowledge and conservation in Kalimantan, Indonesia 1. Econ Bot 52 (3), 229-250.

Canales M, Hernandez T, Caballero J, Romo de Vivar A, Avila G, Duran A, Lira R. 2005. Informant consensus factor and antibacterial activity of the medicinal plants used by the people of San Rafael Coxcatlan, Puebla, Mexico. J Ethnopharmacol 97: 429-439.

Chan KL, Choo CY, Abdullah NR, Ismail Z. 2004. Antiplasmodial studies of Eurycoma longifolia Jack. using the lactate dehydrogenase assay of Plasmodium falciparum. J Ethnopharmacol 92 (2): 223-227.

Cocks ML, Dold AP. 2006. Cultural significance of biodiversity: the role of medicine plant in urban african cultural practices in the Eastern Cape, South Africa. J Ethnobiol 26 (1): 69-78.

Dalle SP, Potvin C. 2004. Conservation of useful plants: an evaluation of local priorities from two indigenous communities in Eastern Panama. Econ Bot 58: 138-57.

Eyssartier C, Ladio AII, Lozada M. 2008. Cultural transmission of traditional knowledge in two population of North-western Patagonia. J Ethnobiol Ethnomed 4 (25): 1-8.
Farouk AE, Benafri A. 2007. Actibacterial activity of Eurycoma longifolia Jack a Maleysian medicinal plant. Saudi Arabia Med J 28 (9): 14221424.

Giovannini P, Reyes-García V, Waldstein A, Heinrich M. 2011. Do pharmaceuticals displace local knowledge and use of medicinal plants? Estimates from a cross-sectional study in a rural indigenous community, Mexico. Soc Sci Med 72 (6): 928-936.

Guimbo ID, Muller J, Larwanou M. 2011. Ethnobotanical knowledge of men, women and children in rural Niger: A mixed methods approach. Ethnobot Res Appl 9: 235-242.

Heinrich M, Ankli A, Frei B, Weimann C, Sticher O. 1998. Medicinal plants in Mexico: healers' consensus and cultural importance. Soc Sci Med 47: 1859-1871.

Helida A, Zuhud EAM, Hardjanto, Purwanto, Hikmat A. 2015. Index of cultural significance as a potential tool for conservation of plants diversity by communities in the Kerinci Seblat National Park. Jurnal Manajemen Hutan Tropika 21 (3): 192-201.

Hoffman B, Timothy. 2007. Importance indices in ethnobotany. Ethnobot Res Appl J 5: 201- 218.

Homerverge GO, Young-Dong K. 2014. Quantitative ethnobotanical study of the medicinal plants used by the Ati Negrito indigenous group in Guimara sisland, Philippines. J Ethnopharmacol 157 : 228242.

Ju Y, Zhuo J, Lui B, Long C. 2013. Eating from the wild: diversity of wild edible plants used by Tibetans in Shangri-la-region, Yunnan, China. J Ethnobiol Ethnomed 9 (28): 1-22.

Kashio M, Johnson DV. 2001. Monograph on benzoin (balsamic resin from Styrax species). FAO Regional Office for Asia and The Pasific, Bangkok

Keller GB, Mandiga H, Maass BL. 2005. Diversity and genetic erosion of traditional vegetables in Tanzania from the farmer's point of view. Plant Genetic Resources: Characterization and Utilization 3: 400-413.

Kuo PC, Shi LS, Damu AG, Su CR, Huang CH, Ke CH, Wu JB, Lin AJ, Bastow KF, Lee KH, Wu TS. 2003. Cytotoxic and antimalaria $\beta_{-}$ Carboline alkaloids from the roots of Eurycoma longifolia. J Nat Prod 66 (10): 1324-1327.

Kusters K, Belcher B. 2004. Forest Products, Livelihoods and Conservation. Volume 1-Asia. Center for International Forestry Research, Bogor.

Kvist PL, Andersen MK, Stagegaard J, Hesselsoe M, Llapapasca C. 2001. Extraction From Woody Forest Plants in Flood Plain Communities in Amazonian Peru: Use, Choice, Evaluation and Conservation Status of Resources. For Ecol Manag 150: 147-174

Langenheim JH. 2003. Plant Resins. Timber Press, Hong Kong.

Lopez C, Shanley P. 2004. Kekayaan Hutan Asia. Makanan, RempahRempah, Kerajinan Tangan, dan Resin. Center for International Forestry Research (CIFOR), Jakarta. [Indonesian]

Martin GJ. 1995. Ethnobotany a people and plants conservation manual. Chapman and Hall. London, UK.

Menendez-Baceta G, Aceituno-Mata L, Reyes-García V, Tardío J, Salpeteur M, Pardo-de-Santayana M (2015) The importance of cultural factors in the distribution of medicinal plant knowledge: Acase study in four Basque regions. J Ethnopharmacol 161: 116-127.

Nasution J. 2009. Oukup. Karo Traditional Herb for Postnatal Health: An Analysis of Bioprospecting in Tropical Vegetation of Indonesia. Indonesia. [Thesis]. Departement of Biology, Bogor Agricultural University. [Indonesia]

Ong G, Kim YG. 2014. Quantitative ethnobotanical study of the medicinal plants used by the Ati Negrito indigenous group in Guimaras island, Philippines. J Ethnopharmacol 157: 228-242.

Pei S, Zhang G, Huai H. 2009. Application of traditional knowledge in forest management: ethnobotanical indicators of sustainable forest use. For Ecol Manag 257: 2012-2017.

Perales H, Benz B, Brush S. 2005. Maize diversity and ethnolinguistic diversity in Chiapas, Mexico. Proc Nat Acad Sci USA 102 (3): 949954.

Phillips O, Gentry AH. 1993. The useful plants of tambopata, peru: i. statistical hypotheses tests with a new quantitative technique. Economic Botany 47: 15-32.

Prance GT, Balee W, Boom BM, Carneiro RL. 1987. Quantitative ethnobotany andthe case for conservation in Amazonia. Conserv Biol 1: 296-310.

Phillips O. 1996. Some quantitative methods for analyzing ethnobotanical knowl-edge. In: Alexiades MN, Sheldon JW (eds.). Selected Guidelines for Ethnobotanical Research: A Field Manual. New York Botanical Garden, New York. 
Pieroni A. 2001. Evaluation of the cultural significance of wild food botanicals tra-ditionally consumed in Northwestern Tuscany, Italy. J Ethnobiol. 21: 89-104.

Purba EC, Nisyawati, Silalahi M. 2016. The ethnomedicine of the Batak Karo Peoples of Merdeka Subdistrict, North Sumatra, Indonesia. Intl J Biol Res 4 (2) : 181-189.

Quinlan M, Quinlan R. 2007. Modernization and medicinal plant knowledge in a Caribbean horticultural village. Medical Anthropol Quarterly 21:169-192.

Ragupathy S, Steven NG, Maruthakkutti M, Velusamy B, Ul-Huda MM 2008. Consensus of the 'Malasars' traditional aboriginal knowledge of medicinal plants in the Velliangiri holy hills, India. J Ethnobiol Ethnomed 4 (8): 1-14.

Signorini MA, Piredda M, Bruschi P. 2009. Plants and traditiona knowledge: An ethnobotanical investigation on Monte Ortobene (Nuoro, Sardinia). J Ethnobiol Ethnomed 5 (6): 1-14.

Silalahi M. Supriatna J, Walujo EB, Nisyawati. 2013. Local knowledge and diversity of medicinal plants in sub-ethnic Batak Karo, North Sumatra. Proceeding of The National Seminary Biodiversity and Indonesia Tropica Ecology. Padang. [Indonesian]

Silalahi M. 2014. The Ethnomedicine of the Medicinal Plants in Subethnic Batak North Sumatra and the Conservation Perspective. [Dissertation]. Departement of Biology, University Indonesia [Indonesia].

Silalahi M, Nisyawati, Walujo EB., Supriatna J. 2015a. Local knowledge of medicinal plants in sub-ethnic Batak Simalungun of North Sumatra, Indonesia. Biodiversitas 16 (1): 44-54.

Silalahi M. 2016. Ecology of medicinal plants of agroforest in Surung Mersada Village, Phakpak Bharat Distict, North Sumatra. Jurna Biologi 19 (2): 89-94.

Silalahi M, Nisyawati, Walujo EB, Supriatna J, Mangunwardoyo W. 2015b. The localknowledge of medicinal plants trader anddiversity of medicinal plants in the Kabanjahe traditional market, North Sumatra, Indonesia. J Ethnopharmacol 175: 432-443.

Silva VA, Andrade LHC, Albuquerque UP. 2006. Revising the cultural significance index: the case of the Fulni-ô in northeastern Brazil. Field Methods 18: 98-108.

Srithi K, Balslev H, Wangpakapattanawong P, Srisanga P, Trisonthi C 2009. Medicinal plant knowledge and its erosion among the Mien (Yao) in northern Thailand. J Ethnopharmacol 123: 335-342.

Statistika Desa Peadundung. 2012. Tatistika Desa Peadundung, Kecamatan Pakkat, Kabupaten Humbang Hasundutan, Sumatera Utara. [Indonesian]
Sujarwo W, Arinasa IBK, Salamone F, Caneva G, Fattorini S. 2014. Cultural Erosion of Balinese Indigenous Knowledge of Food and Nutraceutical Plants. Econ Bot 68 (4): 426-437.

Sujarwo W, Caneva G. 2016. Using quantitative indices to evaluate the cultural importance of food and nutraceutical plants: Comparative data from the Island of Bali (Indonesia). J Cult Heritage 18: 342-348.

Sujarwo W, Caneva G. 2015. Ethnobotanical study of cultivated plants in home gardens of traditional villages in Bali (Indonesia). Human Ecol 43 (5): 769-778

Talbott SM, Talbott JA, George A, Pugh M. 2013. Effect of tongkat ali on stress hormones and psychological mood state in moderately stressed subjects. J Intl Soc Sports Nutr 10 (28): 1-7.

Tardío J, Pardo-de-Santayana M. 2008. Cultural importance indices: a comparative analysis based on the useful wild plants of Southern Cantabria (Northern Spain). Econ Bot 62: 24-39.

Thomas E, Vandebroek I, Sanca S, Van Damme P. 2009. Cultural significance of medicinal plant families and species among Quechua farmers in Apillapampa, Bolivia. J Ethnopharmacol 122: 60-67.

Torre-Cuadros MA, Islebe GA. 2003. Traditional ecological knowledge and use of vegetation in southeastern Mexico: a case study from Solferino, Quintana Roo. Biodiv Conserv 12: 2455-2476.

Turner NJ. 1988. "The importance of a rose": evaluating the cultural significance of plants in Thompson and Lillooet Interior Salish. Amer Anthropol 90: 272-290.

Vandebroek I, Calewaert J, De Jonckheere S, Sanca S, Semo L, Van Damme P. 2004. Use of medicinal plants and pharmaceuticals by indigenous communities in the Bolivian Andes and Amazon. Bull World Health Organization 82 (4), 243-250.

Vitalini S, Iriti M, Puricelli C, Ciuchi D, Segale A, Fico G. 2013. Traditional knowledgeon medicinal and food plants used in Val San Giacomo (Sondrio, Italy) - an alpine ethnobotanical study. J Ethnopharmacol 145: 517-529.

Voeks RA. 2007. Are women reservoirs of traditional plant knowledge? Gender, ethnobotany and globalization in northeast Brazil. Singapore J Trop Geogr 28: 7-20.

www.ThePlantlist. 2017. The Plantlist Database. www.theplantlist.org. (accessed 04.04.17).

Xavier TF, Kannan M, Lija L, Auxillia A, Rose AKF, Kumar SS. 2014 Ethnobotanical study of Kani tribes in Thoduhills of Kerala, South India. J Ethnopharmacol 152: 78-90. 